\title{
The Effectiveness of Implicit Brand Placement in Online Videogame Streaming Services: An Eye Tracking Study
}

\author{
Vincent Duhaime \\ Université du Québec à Montréal \\ Sandrine Prom Tep \\ Université du Québec à Montréal \\ Manon Arcand \\ Université du Québec à Montréal
}

Widely studied in traditional advertising contexts, research on brand placement is still scarce on videogame streaming platforms such as Twitch. These environments combining video games and social media, this article investigates the impact of product placement on branding using eye tracking. Similar to Twitch content, a video was made of a professional gamer's streaming session with Hearthstone. The video was randomly presented with or without product placement to 40 subjects, followed by a questionnaire. The results demonstrate that, it captures the attention of viewers, which positively affects unaided and aided brand recall, and that both positively influence attitude towards the brand.

Keywords: product placement, videogame streaming, eye tracking, attention, recall, attitude toward the brand, brand recall

\section{INTRODUCTION}

The appetite of young gamers for watching players perform live shows no sign of waning on Twitch, a platform which specializes in live streaming video game sessions by individual streamers. This industry has grown tremendously in recent years, from 192 to 355 billion viewing minutes between 2014 and 2017 (Twitch, 2018) In 2014, it already ranked 4th among the websites with the most traffic in the United States according to the Wall Street Journal (Twitch, 2018) in 2018 the site reached an average of 15 million users per day (Andronico, 2018). According to Twitch (Twitch, 2017), the audience is predominantly male $(81 \%)$ and between 18 and 34 years of age $(55 \%)$, which explains why more than a third of traffic on the platform is through a mobile device (Gotter, 2017). The growing popularity of this type of medium therefore offers an attractive point of contact for brands aiming at consumers less than 35 years of age, particularly as they are moving away from television (Nielsen, 2017). These emerging hybrid social platforms compete with audiences equal in size to those using traditional media thus offering new opportunities for monetization. On Twitch, the game is broadcasted in full screen, however the streamer or broadcaster playing the game appears facing the camera, in a small rectangle placed in a 
corner of the frame, to comment on his actions. A chat enables viewers to encourage the streamer, or to offer advice. Viewers both can therefore focus on the game itself, and also listen to, see and interact with the player and other viewers. In summary the medium combines gaming, video, and the social web, in which the consumer is both creator and consumer of content (prosumer) according to a spectrum of activity that can be modulated between the two extremes (Sjöblom, et.al., 2019).

In marketing research, the positive effect of product placement on brand recall and recognition has been widely demonstrated in traditional media such as film or television (Gregorio, \& Sung, 2010; Lehu, 2005; Stern, \& Russell, 2004), or video games (Glass, 2007; Hang, 2014; Lee, \& Faber, 2007; Nelson, 2002) even when implicit (Hang, 2014). However, despite this consumer infatuation with live streaming of video games on Twitch, and the fact that product placement is ubiquitous there since it is the main source of revenue for streamers (Hamilton, et.al., 2014), few studies have been conducted to measure the effectiveness of brand placement on consumer behavior in this new type of medium. Gangadharbatla (2016) shows, however, that an individual who watches someone else play video games (passive gamer) can recall four times more brands than the player (active gamer). Moreover, the author argues that the best way to understand how a consumer behaves towards brands encountered in a specific environment is to measure his/her behavioral responses directly, including recall measures. Indeed, since the player's attention is devoted mainly to the tasks required of the game, product placement in a video gaming context is a priori more difficult for the gamer (consumer) to notice (Lee, et.al., 2007), especially when implicit or peripheral (Schneider, et.al., 2005; Russell, 2002) given the player's reduced attention already occupied by the game (Kahneman, 1973).

Thus, the purpose of this article is to measure the effect of implicit brand product placement in a video game stream on brand recall and recognition. The effect of brand recall on attitude towards the brand and intention to purchase are also measured. While several aim to measure the direct effect of brand placement on brand recall (Lee, \& Faber, 2007; Nelson, 2002; Schneider, et.al., 2005; Law, \& Braun, 2000), this study stands out by going beyond the traditional measure of recall, in that attention paid to product placement is measured by direct behavioral observation (eye tracking). This study addresses the following question: Does the theoretical knowledge associated with brand placement and its impact in traditional media also extend to video game streaming? The answer to this question enables the formulation of recommendations for advertisers and marketing managers wishing to monetize and include these platforms in their media portfolio.

\section{LITERATURE REVIEW}

\section{Social Commerce and Live Streaming Services}

For decades now, Internet users have been used to social media services such as Twitter, Facebook to name only a few of the well-known ones. In more recent years, social video sharing platforms have emerged such as Twitch and Youtube Live. This type of social video content creation is called livestreaming and "can refer to many aspects of digital technology and culture, ranging from purely technical data transfer, to the cultural phenomenon of streaming" (Sjöblom, et.al., 2019, p.21). "While such activity is often waived merely as a manifestation of video game culture and an extension of online behavior by adolescents, the phenomenon has begun to generate significant revenue and has managed to shift media consumption behavior from large commercial organisations towards content created by private individuals" (p.20); the authors add that this type of professionally-generated user content becomes mainstream and is supported with social features such as "the chat window; the ability to follow and share a stream or a streamer; some revenue features such as the subscribe button partner program" (p.21). So, out of the good old traditional social media with their now well-established type of digital media prosumption (i.e. digital media consumption and production activities merged altogether), has emerged this unique and new form of social commerce referred to as "a special combination of multiple media forms" such as live streaming and chat rooms for user communication. According to $\mathrm{Hu}$ et al. (2017, p.595) "the nature of live video streaming activity not only offers a real-time watching experience for audiences, but also provides opportunities to communicate and socialize among broadcasters and other 
co-viewers". The authors use social identity theory to explain the increasing propensity of massive audiences for consuming streaming services and define the "para social interactions" taking place in this cyber context between streamer and audience, as well as among audience viewers themselves as they are co-sharing the experience. "Specifically, the entirety of live streaming content is not merely provided by broadcasters alone; the contributions from multi-type audiences complement the whole experience of watching streaming contents and differentiate live streaming from consuming traditional television programs." (p.597). As far as the digital realm is concerned, it is fairly common place to say that academic research falls behind practice, and it is the specific aim of this research to try and remedy this. In paying attention to brand placement in such novel s-commerce environments, which are offering a higher engaging and interactive consumption context than standard social media platforms, our research differentiates itself from well-studied brand product placement contexts in media types such as film, short videos or television programs.

\section{Product Placement in Video Games and New Social Platforms}

Brand placement can be defined as the paid, planned and non-intrusive introduction of selected brands in a media program for the purpose of influencing consumers (Karrh, 1998). Studies demonstrating its effectiveness on brand recall, purchase intention and attitudinal change have been conducted in the video games field (Glass, 2007; Lee, \& Faber, 2007; Nelson, 2002) and more recently on streaming platforms (Gangadharbatla, 2016; Da Silva, \& Cléret, 2012) and e-sports (Hallmann, \& Giel, 2017). Hallman and Giel (2017) consider that product placement as a promotional tool is particularly relevant in the context of the new sport category of video game competitions (e-sports), since in general, product placement in video games is perceived positively by the player (Lehu, \& Bressoud, 2008).

\section{The Effect of Product Placement on Attention}

Attention is defined as a measure of brain processing activity devoted to a specific stimulus (Solomon, et.al., 2013) or the allocation of mental, visual or cognitive resources on an object (Texeira, 2014). In a promotional context, the greater the attention of the consumer, the more the message will have an impact on the latter (Texeira, 2014). Capturing the consumer's attention is therefore a primary objective of product placement, and the reason why measuring this attention precisely and directly via eye tracking, based on behavior and not on reported measurements, represents methodological added value to existing product placement studies in these emerging environments.

\section{Characteristics of Brand Product Placement}

The size, central placement (Lehu, 2005) and liveliness of colors (Solomon, et.al., 2013) of the stimulus, are elements that capture the consumer's attention. In this study, the size used for the product placement (stimulus) is small, the black and green colors provide a fairly sharp contrast, and the location of the stimulus is peripheral at the bottom of the screen on the far left. Argan et al. (2007) state that a product placement must be present for a period of time long enough to capture attention, without annoying the consumer. It has also been shown that the use of product placement in a video game can sometimes be less obvious to the player whose attention is devoted mainly to tasks in the game rather than the product placement. It is therefore necessary that the brands presented be placed on the screen such that the player can notice them (Lee, \& Faber, 2007). When product placement is implicit, Schneider et al. (2005) and Russell (2002) show it still captures attention, but that it is less effective, especially for brand recall, than explicit product placement. Although no study has specifically tested the effect of product placement on attention in the context of video game streaming, based on the various studies that have investigated the effect of product placement on attention (Lee, \& Faber, 2007; Argan, et.al., 2007; Boerman, et.al., 2015), the following hypothesis is proposed:

H1: The use of implicit brand placement has a positive effect on the attention drawn to the product in the area of interest in video game streaming. 


\section{The Effect of Product Placement on Brand Recall}

Brand awareness is the totality of what exists in the consumer's mind with regards to the brand (Keller, \& Lehmann, 2003), namely the thoughts, images and perceptions associated with a brand in the consumer's memory. For brand placement to be considered effective, the consumer must remember the brand (Williams, et.al., 2011). In fact, the primary goal of branded product placement is to increase brand recall (unaided and aided) ${ }^{1}$ (Karrh, et.al., 2003).

Brand recall (unaided) is defined as the consumer's ability to correctly remember the name of a brand when the product category is mentioned, and no additional clues provided (Keller, 1993). The effectiveness of product placement in a cinematographic work on brand recall has already been demonstrated (D'Astous, \& Chartier, 2000). Brand placement in video games appears to have a similar impact in a movie (Nelson, 2002; Schneider, et.al., 2005). Mackay et al. (2009) even point out that brand placement in video games can generate brand recall among people who do not have any positive predisposition towards the brand. Brand recognition (aided recall) is defined as the ability of a consumer to correctly differentiate between a brand to which he/she has already been exposed and another brand (Keller, 1993). Aided recall is positively influenced by brand placement in a film (Williams, et.al., 2011; D'Astous, \& Chartier, 2000). Viewers can also distinguish brands that have appeared on screen from those which were not presented in a film (Babin, \& Carder, 1996).

Brand placement also has a positive influence on brand recognition when viewing a video on Youtube, even if it was implicitly embedded in it (Verhellen, et.al., 2013). In fact, whether the brand placement is prominent or unobtrusive, a consumer who views a video on YouTube recognizes the brands that are embedded in it much better than other brands that are not presented (Verhellen, et.al., 2013). Based on this literature, it is proposed that the effectiveness of brand placement will translate to the video game streaming context. The following hypothesis is proposed:

H2: The use of implicit brand placement in video game streaming has a positive effect on unaided and aided brand recall.

\section{The Effect of Attention on Brand Recall}

For product placement to result in brand recall, the product must be noticed by the consumer. Since his/her attention is limited (Kahneman, 1973), it is important to evaluate the allocation of the consumer's attention resources when exposed to brand placement. To our knowledge, very few brand placement studies have included a measure of viewer attention, with most focusing on the direct effect of product placement on brand recall and recognition. Boerman et al. (2015) demonstrate however that when attention is drawn to a product placement in a television program, an increase in brand recall follows. Similarly, the positive impact of attention on brand recall has been demonstrated in the context of banner advertising (Sajjacholapunt, \& Ball, 2014). Based on the results of the various research studies mentioned above, the following hypothesis is proposed:

H3: Attention placed on a branded product placement has a positive effect on unaided and aided brand recall in video game streaming.

\section{The Effect of Brand Recall on Attitude Towards a Brand}

Attitude is defined as a disposition to respond favorably or not to an object (Ajzen, 2005). For Keller (1993) building an attitude towards a brand is directly related to awareness of it. In general, good awareness of a brand in a product placement context leads to a more favorable attitude, or even a change in perception of the brand (Srivastava, 2015). Recall of a brand presented in a medium can occur explicitly or implicitly (Law, \& Braun, 2000). Indeed, even if a consumer does not explicitly remember a brand that he/she saw during a product placement (unaided recall), familiarity with the logo having been exposed to it in the media (aided recall) positively influences his/her attitude towards the brand in question (Law, \& Braun, 2000). In both cases, memorization affects attitude towards the brand (Russell, 2002; Homer, 2009). 
According to Russell (2002), brand recall induced by a product placement can have either a positive or negative impact on attitude depending on its manner of presentation. For example, if seen as too prominent, in that its use for promotional purposes is obvious and unrelated to the narrative, brand recall may be strong, but have a negative impact on attitude towards the brand. Conversely, this relationship will be positive if the product placement is coherent, and adds value to the story (Russell, 2002). In the context of video games, Mackay et al. (2009) have shown that product placement has an influence on brand awareness that results in a change in attitude towards the brand, even among players whose attitude towards the brand was somewhat weak before the product placement. Having played a video game in which several brand placements were included, gamers show a significantly higher awareness of the brands presented - in comparison to brands in the same product category which were not presented. Similarly, Glass (2007) showed that brand recall is linked to a more positive appraisal of the brand used in the video game. However, Gibson et al. (2014) mention that awareness of a brand leads to a positive attitude towards the placed product provided that the consumer does not have the impression of an attempt to be persuaded to favour the brand. Contrariwise, the impact on brand awareness will have a negative effect on brand attitude. Note that product placement used in this study is implicit and discreet, such that it should not be perceived as having the objective of persuading the consumer to adhere to the brand.

In spite of the growing popularity of streaming, especially video games, which constitutes a new medium in its own right, and with distinct business models specific to s-commerce, the corresponding marketing research is still insufficient; it does not enable a product placement's impact of brand awareness on attitude to be extended to this emerging context. The following hypothesis is therefore proposed:

H4: Brand recall (whether unaided or aided) has a positive effect on attitude towards the brand presented in video game streaming.

\section{The Effect of Attitude on Purchasing Intention}

Attitude towards the brand is often the primary factor influencing the consumer's choice of one brand over another (Keller, 1993). The close positive connection between attitude and purchase intention is well established in the literature (Keller, 1993; Shimp, 1981; Spears, \& Singh, 2004). For millennials, when brand placement has a positive impact on brand attitude, this increase in positive attitude translates to a higher purchase intention towards the product presented and viewed in a TV show or film (Kit, \& P'ng, 2014). On YouTube, product placement in videos created by influencing YouTubers positively affects attitude towards the product, and this positive attitude translates into purchase intentions (Verhellen, et.al., 2013). On the other hand, in this context, the products explicitly endorsed by the YouTuber elicit a stronger response in terms of attitude towards the brand and purchase intention, in comparison with the products that simply appear on the screen without any mention (Verhellen, et.al., 2013). According to Adis and Jun (2013), in the video game universe, brand placement generates a more favorable attitude towards the placed brand which is accompanied by an increase in purchase intentions for the brand product.

However, despite the considerable number of studies that have examined purchase intentions in a product placement context, the attitude-purchase intention relationship has not been confirmed in the streaming medium as such. It is therefore possible to formulate the following hypothesis:

H5: Brand attitude has a positive effect on purchase intention when the brand is placed in videogame streaming. 


\section{FIGURE 1 \\ CONCEPTUAL FRAMEWORK AND RESEARCH HYPOTHESES}

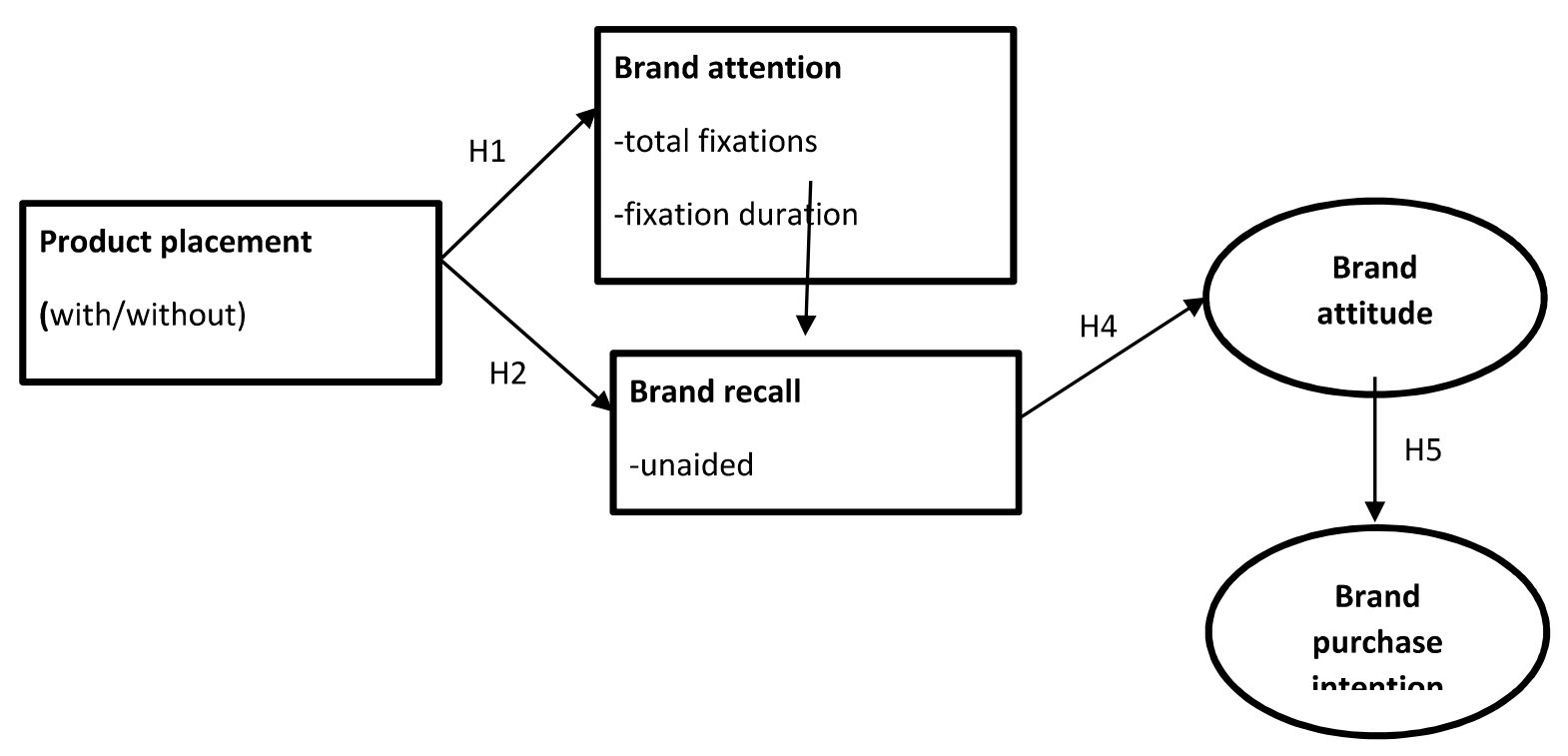

\section{METHODOLOGY}

To test the research hypotheses, a single-factor two-group experimental design was used as it is a common methodology for testing the effectiveness of product placement on variables of interest (Lee, \& Faber, 2007; Gangadharbatla, 2016). The experiment was conducted in a laboratory to limit the influence of external variables and thus maximize the internal validity of the research (Creswell, 2013).

Independent variable "product placement" was manipulated at two levels (with or without product placement). The other variables were measured: attention to product placement, brand recall, brand attitude and purchase intention. To do this, a video game stream was created for the study with the help of a professional gamer. The video features a player who explains how to create a specific character class while facing another player in an online game of Hearthstone ${ }^{2}$. To maximize the realism of the experiment, the video is faithful to those broadcasted on Twitch: the current game is shown full-screen with the active player in a smaller superimposed window located in the lower left corner of the screen. In addition to broadcasting live images from the video game, the player provides advice for the audience. A product placement is incorporated into the video on the far left (in the "with placement" condition) in the spot where the player is shown, it is visible throughout the video. The player does not interact with the product placement in question, it is implicit in nature. A can of Monster energy drink was chosen for the product placement in the experiment. This choice was justified by an existing association between energy drinks and video gamers. In fact, gamers who consume energy drinks more than once a week spend an average of 4 additional hours playing video games compared to those who consume one or less per week (Larson, et.al., 2014). The video stream viewed by the subjects in the "without placement" condition is identical to that described above, except that the Monster can is missing.

Forty subjects participated in the study who were randomly assigned to one of the two conditions (with/without product placement). To be eligible for the study, the subject had to be an adult, possess a mobile device, and play video games for at least 1 hour per week. A \$10 compensation was offered, and the experimental procedure lasted for an average of twenty minutes. The subjects were recruited via Facebook, online gaming communities, and through an announcement to students on the university campus. Finally, the sample comprised mostly males (93\%) with $85 \%$ of them aged between 21 and 29 , which is close to the audience profile for video game streams broadcast on Twitch. In addition, $52 \%$ had reached at least college level education. We note that $80 \%$ of the subjects are familiar with the game 
Heartstone and that $72 \%$ of them watch video game streams, spending on average more than $10 \mathrm{hrs} /$ week (685 minutes) playing video games. The experimental groups are similar on all these variables $(\mathrm{p}>0.05)$ except for schooling, where the group "without product placement" is significantly $(p<0.05)$ more educated than the "with product placement" group.

\section{Conduct of Experimentation and Measurement of Variables}

The experiment was conducted during the summer of 2017. Once the subject had arrived at the laboratory, the researcher explained what the task entailed and invited him/her to sign the consent form. When these steps were completed, the researcher calibrated the eye tracking glasses ${ }^{3}$. The subject was then randomly assigned with one of two conditions and the video to be fully viewed on the Apple Ipad Mini tablet, before responding to a post-experiment questionnaire. As already mentioned, the choice of mobile device is justified by the fact that its use already represents more than a third of the views on Twitch, and that it is the preferred choice of young people under 35 for activities such as instant messaging (96\%), video games $(74 \%)$ social networks (69\%) according to eMarketer (Segal, 2018). These three activities are now combined in video game streaming services platforms as a new social medium. Finally the choice of a mini tablet measuring $7.9^{\prime \prime}$ is justified by the fact that phablets $(5.0 "-6.9$ "), the neologism to designate smartphones almost the size of a mini tablet ( $7.0 "-8.4$ "), are becoming increasingly popular with consumers according to Flurry Analytics (2015). Eye tracking technology was used to measure the attention paid to product placement. The system captures data at a refresh rate of 60 $\mathrm{Hz}$, i.e. the number of times a fixation is measured per second. This sampling rate is common and scientifically accepted in the collection of valid data (Quinn, et.al., 2007). An area of interest was defined in the region of the Monster can in both versions of the video. The software counts all eye fixations located in the area of interest for each subject. Two metrics were used to evaluate the attention to product placement: the number of total fixations recorded in the area of interest (the location of the product placement when present) and the total sum of the duration of all fixations on the area of interest while viewing the video. The total number of recorded fixations is an indicator of attention since visual information is assimilated by the brain whenever there are fixations (Wolverton, \& Zola, 1983). The total duration of fixations on a stimulus also measures attention paid to the stimulus in question (Pernice, \& Nielsen, 2009). These measures are used in research presenting a specific area of interest whether a product placement be in a video game (Herrewijn, \& Poels, 2013), television (Boerman, et.al., 2015) or the location of a link on Google (Mediative, 2014). Eye tracking has proven itself as a tool for measuring attention which is effective and accurate (Mackay, et.al., 2009; Herrewijn, \& Poels, 2013; Mediative. 2014). To measure brand recall, standard unaided and aided recall procedures were used. The questions: "Did you see brands in the video? Yes, No? ", and "If so, can you name one? ", served as a measure of unaided recall. For aided recall, ten brands were presented (photo and logo) to the subjects. They were asked to check all the brands noticed in the video. To measure the subject's attitude towards the Monster brand, a 5-point differential semantic scale (4 items, i.e. Good / bad, like / hate) elaborated by Goldsmith (2002) were employed. Finally, for the purpose of measuring purchase intention, the Baker and Churchill (1977) scale was used (Likert 5 points, 3 items).

\section{Pretests and Manipulation Tests}

A pretest was conducted to validate the manipulation (i.e., that the video was considered realistic in comparison to those found on Twitch), and that the product placement was observable in the "with placement" condition (and vice versa). Each version of the video (with and without placement) was viewed by 10 people for a total of 20 people. The following question was posed after the viewing: $I$ noticed one or more brands in the video (5-point Likert scale, where $1=$ not at all and $5=$ absolutely). The results of the pretest are conclusive. The ANOVA indeed indicates a significant difference between the two groups as to perception of the presence of a brand $\left(\mathrm{M}_{\text {with }}=4.2\right.$ and $\left.\mathrm{M}_{\text {without }}=1.1, \mathrm{p}<0.01\right)$. The realism of the created video game stream was pre-tested with the following question: I find that the video game stream looks like those that I see on YouTube or Twitch (Likert Scale on 5 points where $1=$ not at all and $5=$ absolutely). The results of the ANOVA show that there is no significant difference between 
the two groups $\left(\mathrm{M}_{\text {with }}=4.4\right.$ and $\left.\mathrm{M}_{\text {without }}=4.7, \mathrm{p}>0.05\right)$ and that the subjects find that the video realistically represents this context (average of $4.5 / 5$ ).

\section{RESULTS}

\section{Descriptive Results With Reliability and Validity of Measures}

To ensure the psychometric quality of the scales used to measure attitude towards the brand and purchase intention, principal component factor analyses and the calculation of the Cronbach alphas were performed. Note that all factor scores are greater than 0.70 , well above the threshold of 0.50 recommended by Hair et al. (2010) for satisfactory convergent validity. The Cronbach alphas are well above the 0.70 threshold recommended by Nunnally (1978). Given the good psychometric quality of the scales, mean scores were calculated for the study constructs to perform hypothesis tests. We note that on average attitude towards the Monster brand is $3.23 / 5$ and that purchase intention is rather low at $2.2 / 5$. The average number of fixations per subject was 550 for the total duration of the video.

To test the effect of product placement on attention (H1), one-sided t-tests were performed on both attention indicators. In agreement with $\mathrm{H} 1$, the results of the t-tests indicate that attention to the area of interest is significantly higher when the placement is present, both for total fixation duration (Mwith = $0.60 \mathrm{sec}$. vs Mwithout $=0.14 \mathrm{sec} ., \mathrm{p}<0.05)$ and for the number of total fixations (Mwith $=1.45$ and Mwithout $=0.45, p<0.05)$, corresponding to moderate effects $(\mathrm{y}=0.32$ and 0.30 respectively). It can therefore be stated that product placement positively influences attention paid to a defined area of interest thus confirming $\mathrm{H} 1$. $\mathrm{H} 2$ proposes that product placement also positively influences brand recall, i.e. unaided and aided recall. Cross-tabulation and chi-square (one-sided) results confirm H2. Indeed, for unaided recall, no subject in the 'without product placement' condition spontaneously identified the Monster brand while half of the subjects who viewed the video 'with' product placement were able to name it unaided. This relationship is significant (chi-square $=13.33, \mathrm{p}<0.01$ ) and strong (Cramer $\mathrm{V}=$ 0.57). Similar results were obtained for aided recall, given that only one subject of the 'without product placement' condition said that he recognized Monster and that half of the subjects in the 'with product placement' condition spotted it (chi square $=10.16, \mathrm{p}<0.01$, Cramer $\mathrm{V}=0.50$ ). It can therefore be concluded that $\mathrm{H} 2$ is confirmed. $\mathrm{H} 3$ proposes that attention influences the level of brand recall. A logistic regression was carried out to test this hypothesis by incorporating total fixation duration and the manipulated variable (product placement) as predictors, with aided recall $(1=$ yes, $0=$ no $)$ as the dependent variable. The model explains $55 \%$ of the total variance (Nagelkerke's R2) and correctly classifies $85 \%$ of the subjects. The results demonstrate that the odds ratio of recognizing the brand increases significantly and strongly for subjects in the condition with product placement $(\operatorname{Exp}(\mathrm{B})=$ $13.68, \mathrm{p}<0.05)$. In fact, the chance of recognizing the brand is therefore 13.68 times greater for the subjects assigned to the condition with product placement, compared to those in the reverse condition. Similarly, attention to the area of interest enables brand recognition to be predicted significantly (Exp (B) $=7.98, \mathrm{p}<0.05)$. Hence for each additional second of attention, the chance of recognizing the brand increases by almost $800 \%$. A logistic regression using the number of total fixations instead of the fixation time duration shows similar results (Nagelkerke's $\mathrm{R} 2=0.56$, odds ratio number of total fixations $=2.18$, $\mathrm{p}<0.05$, odds ratio for the condition with product placement $=15.34, \mathrm{p}<0.05)$. H3 is therefore confirmed.

Brand recall should positively influence attitude towards the brand: $\mathrm{H} 4$ proposes this. The results of (one-sided) t-tests do support this hypothesis but only marginally. Indeed, the subjects who were able to name the brand spontaneously (yes) display a more positive attitude (Myes $=3.7$ vs Mno $=3.07$ ) but this difference is only marginally significant $(\mathrm{p}=0.08)$. The same goes for the effect of aided recall (Myes $=$ $3.68 \mathrm{vs} \mathrm{Mno}=3.05, \mathrm{p}=0.08$ ). Hence hypothesis H4 is marginally confirmed. Finally, as predicted by H5, attitude towards a brand has an impact on purchase intention. The results of the regression are conclusive $(\mathrm{F}=45.41, \mathrm{p}<0.01)$ and the relationship is positive $(\mathrm{b}=0.83)$ and very strong $(\mathrm{r}=0.738)$. Attitude towards the brand explains $54.4 \%$ of the variation in the intention to purchase. 


\section{DISCUSSION, LIMITS AND FUTURE RESEARCH}

From a theoretical and methodological point of view, the results of this study make three main contributions to the field of product placement in the context of s-commerce. Firstly, although interest in product placement is common in advertising as a research field, it has not yet addressed this issue in the new media environment of s-commerce platforms. Thus, to the best of our knowledge, it is novel to focus on videogame streaming even though this type of diffusion is becoming more popular to the point of becoming mainstream ( $\mathrm{Hu}$, et.al., 2017), and one of the major contributions of this research is to test the theoretical tenets of traditional media for brand product placement in this new context. The results of our research show that, despite the fact that streaming is a medium distinct from film and television, especially in terms of the social interaction involved in this type of video, which makes it very different from a television show, a movie, a video game and even a music video clip on Youtube, implicit product placement has comparable effects on brand attention, brand recall and attitude towards the brand. A second important contribution lies in the methodology used, namely the use of eye tracking, which enables direct measurement of the attention paid to product placement (vs reported measures using methods such as a questionnaire), a variable often neglected in studies examining the effect of this promotional tool. Eye tracking measures thus validate the key role of attention on brand recall and recognition. The present study further confirms this relationship in a video game stream, extending the theoretical understanding of product placement to this new social medium. Last, but not least, another contribution of the study lies in the device chosen for viewing the video. In fact, participants watched the video game stream on a small mobile device (7.9"), a viewing context comparable to that of more than a third of Twitch's users, reinforcing the external validity of the study. Most research so far has been conducted on large screens (television, cinema or computer screen). However, studies of web-based advertising banners have shown that screen size has a negative effect on click-through rates and conversion rates (Boyle, 2015; Robison, 2014). While other studies have shown that players evaluate a video game more positively when played on a larger screen (Hou, et.al., 2012). The results of the study reveal that the mini tablet screen size, which is rather small in comparison to theaters' screens, television or computer monitors, does not affect the consumer's ability to notice product placements. In conclusion, although no assumptions were made about screen size, the results of the study point to the fact that tablet screen size does not seem to affect the consumer's ability to notice product placements in a streaming context, something which remains to be formally and empirically confirmed in a future study to better address omnichannel offers.

These theoretical contributions also have managerial implications, given the positive externalities on the consumer's memory when exposed implicitly to a brand. This research thus provides several very important elements for a marketing manager wishing to promote a brand using product placement in video game streams, and for streaming platform managers willing to monetize these new highly engaging social media assets. Keep in mind that video live streaming is a favorite option for brands wishing to reach a younger audience since generation $\mathrm{Y}$ is the primary clientele of video game streaming. Indeed, to the extent that the results have demonstrated the positive impact of product placement on a brand when implicitly presented in a video game stream, it is reasonable to conclude that a company that invests in this tool in the context of a video game stream to promote its brand can hope to obtain a substantial return on investment. This added value of product placement is all the greater as this promotional tool is better perceived and more accepted than conventional advertising of a more intrusive nature (Nelson, et.al., 2004). Brand product placement can therefore be used by brands to sustain presence in the minds of consumers or as a complement to a traditional advertising campaign to change attitudes towards the brand and possibly boost sales. The results show, however, that managers must ensure that the product appearing in a video game stream is sufficiently visible to be noticed (in the case of this study, the product placed was bright green), and capture the spectator's attention without surprising him/her, by such means as an insertion of a product relevant to the context, while remaining implicit. Finally, it is interesting to note that the influence of brand attitude on purchase intention has already been demonstrated by several authors (Verhellen, et.al., 2013; Shimp, 1981; Spears, \& Singh, 2004). This study, however, has sought to 
validate this relationship, as modifying purchase intention and consumer behavior are among the ultimate goals of brand placement (Williams, et.al., 2011). The results of the study confirm the positive and strong influence of brand attitude on purchase intention in the context of streaming video game viewing.

This study nevertheless does have some limitations. First, the size of the sample is small, limiting itself to a total of 40 participants only. It is therefore necessary to use caution when generalizing the results. It should also be stressed that the subjects of the two groups differed in their level of education, and that a large majority of recruits for the experiment were male. While this is representative of the target market for platforms such as Twitch, this limitation needs to be noted. Experimentally, the limitation of the laboratory context in which the subjects viewed the video should be mentioned. When viewers consume streaming video content, they do so most of the time in the comfort of their home or on the go. The laboratory, while maximizing the internal validity of the study, does not represent the typical viewing context (even though a mobile device was used). Also, we must mention that the video was watched on a tablet with a 7.9-inch screen, though rather small and close to phablets as we already mentioned, caution is required in generalizing the results in the case of smartphones with smaller screens, knowing that screen size impacts attention (Reeves, et.al., 1999). The limits set out above indicate an equivalent number avenues for future research. For example, it would be relevant to conduct a similar study comparing different devices (computer, tablet, smart phone) to formally compare the impact of screen size on the focus on product placements. Despite these few limitations, the contributions of this study are important and scientifically relevant, and useful also for marketing managers who wish to use video game streams to promote their brand.

\section{ENDNOTES}

1. Aided brand recall is also called brand recognition. Both words are used interchangeably in the text.

2. Heartstone is part of the top 5 games played on Twitch (2015).

3. The authors would like to thank the OPTECH of CTT from CEGEP André Laurendeau who provided the eye tracking glasses, you can find more information about OPTECH on their website: https://www.ccttoptech.ca

\section{REFERENCES}

Adis, A. A. A., \& Jun, K. H. (2013). Antecedents of brand recall and brand attitude towards purchase intention in advergames. European Journal of Business and Management, 5(18), 58-68.

Ajzen, I. (2005). Attitudes, Personality and Behavior (2e éd.). New York, NY: Open University Press. Andronico, M. (2018). Inside the future of Twitch: Watching is the new playing. Resource document. Tomsguide. Retrieved July 10, 2018, from www.tomsguide.com/us/twitch-extentionsonteractive-livestreaming,news-26887.html

Argan, M., Velioglu, M. N., \& Argan, M. T. (2007). Audience attitudes towards product placement in movies: A case from Turkey. Journal of American Academy of Business, 11(1), 161-168.

Babin, L. A., \& Carder, S. T. (1996). Viewers' recognition of brands placed within a film. International Journal of Advertising, 15(2), 140-151.

Baker, M. J., \& Churchill, G. A., Jr. (1977). The impact of physically attractive models on advertising evaluations. Journal of Marketing Research, 14(4), 538-555.

Boerman, S. C., Van Reijmersdal, E. A., \& Neijens, P. C. (2015). Using eye tracking to understand the effects of brand placement disclosure types in television programs. Journal of Advertising, 44(3), 196-207.

Boyle, C. (2015). Mobile apps vs mobile browsers: How usage ad spending and mobile commerce sales by channel. E-marketer. Retrieved July 10, 2018, from https://fr.slideshare.net/ireneventayol/emarketer-mobilecontentactivitiesroundup

Creswell, J. W. (2013). Research Design: Qualitative, quantitative, and mixed methods approaches. Thousand Oaks, CA: Sage publications. 
D'Astous, A., \& Chartier, F. (2000). A study of factors affecting consumer evaluations and memory of product placements in movies. Journal of Current Issues and Research in Advertising, 22(2), 3140.

Da Silva, M.F., \& Cléret, B. (2012). Le placement de produits dans les clips vidéo: une étude exploratoire. Presented in the 11th Journées Normandes de Recherches sur la Consommation: Société et Consommation. Caen, France.

Flurry Analytics. (2015). Stats show phablets continuing their rise to dominance. Retrieved July 10, 2018, from http://mobilesyrup.com/2015/12/29/stats-show-phablets-continuing-their-rise-to-dominance/

Gangadharbatla, H. (2016). A comparison of in-game brand placement for active versus passive players. Journal of Interactive Advertising, 16(2), 117-132.

Gibson, B., Redker, C., \& Zimmerman, I. (2014). Conscious and nonconscious effects of product placement: Brand recall and active persuasion knowledge affect brand attitudes and brand selfidentification differently. Psychology of Popular Media Culture, 3(1), 19-37.

Glass, Z. (2007). The effectiveness of product placement in video games. Journal of Interactive Advertising, 8(1), 23-32.

Goldsmith, R. E. (2002). Explaining and predicting consumer intention to purchase over the internet: An exploratory study. Journal of Marketing Theory and Practice, 10(2), 22-28.

Gotter, A. (2017). Desktop vs. mobile video consumption: What you need to know. Resource Document Business 2 Community. Retrieved July 10, 2018, from

https://www.business2community.com/.video-marketing/desktop-vs-mobile-video-consumptionneed-know-01937184

Gregorio, G., \& Sung, Y. (2010). Understanding attitudes toward and behaviors in response to product placement. Journal of Advertising, 39(1), 83-96.

Hair, J.F., Black, W.C., Babin, B.J., \& Anderso, R.E. (2010). Multivariate Data Analysis (7th edition). Upper Saddle River, NU: Prentice Hall.

Hallmann, K., \& Giel, T. (2017). eSports-Competitive sports or recreational activity? Sport Management Review, 21(1), 14-20.

Hamilton, W. A., Garretson, O., \& Kerne, A. (2014). Streaming on Twitch: Fostering participatory communities of play within live mixed media. In Proceedings of the 32nd annual ACM conference on Human factors in computing systems (pp. 1315-1324). New York, NY: Association for Computing Machinery.

Hang, H. (2014). Brand-placement effectiveness and competitive interference in entertainment media: Brand recall and choice in kids' video-game advertisements. Journal of Advertising Research, 54(2), 192-199.

Herrewijn, L., \& Poels, K. (2013). Putting brands into play: How game difficulty and player experiences influence the effectiveness of in-game advertising. International Journal of Advertising, 32(1), $17-44$.

Homer, P.M. (2009). Product placements: The impact of placement type and repetition on attitude. Journal of Advertising, 38(3), 21-32.

Hou, J., Nam, Y., Peng, W., \& Lee, K. M. (2012). Effects of screen size, viewing angle, and players' immersion tendencies on game experience. Computers in Human Behavior, 28(2), 617-623.

Hu, M., Zhang, M., \& Wang, Y. (2017). Why do audiences choose to keep watching on live video streaming platforms? An explanation of dual identification framework. Computers in Human Behavior, 75, 594-606.

Kahneman, D. (1973). Attention and effort. Englewood Cliffs, NJ: Prentice-Hall.

Karrh, J. A. (1998). Brand placement: A review. Journal of Current Issues and Research in Advertising, 20(2), 31-49.

Karrh, J.A., McKee, K. B., \& Pardun, C. J. (2003), Practitioners' evolving views on product placement effectiveness. Journal of Advertising Research, 43(2), 138-149.

Keller, K.L., \& Lehmann, D.R. (2003). How do brands create value? Marketing Management, 12(3), 2331.

102 Journal of Marketing Development and Competitiveness Vol. 14(3) 2020 
Kit, L. C., \& P'ng, E. L. Q. (2014). The effectiveness of product placement: The influence of product placement towards consumer behavior of the millennial generation. International Journal of Social Science and Humanity, 4(2), 138-142.

Larson, N., DeWolfe, J., Story, M., \& Neumark-Sztainer, D. (2014). Adolescent consumption of sports and energy drinks: Linkages to higher physical activity, unhealthy beverage patterns, cigarette smoking, and screen media use. Journal of Nutrition Education and Behavior, 46(3), 181-187.

Law, S., \& Braun, K. A. (2000). I'll have what she's having: Gauging the impact of product placements on viewers. Psychology \& Marketing, 17(12), 1059-1075.

Lee, M., \& Faber, R. J. (2007). Effects of product placement in on-line games on brand memory: A perspective of the limited-capacity model of attention. Journal of Advertising, 36(4), 75-90.

Lehu, J. M. (2005). Le placement de marques au cinéma : Proposition de la localisation du placement à l'écran comme nouveau facteur d'efficacité potentielle. Décisions Marketing, 37, 17-31.

Lehu, J. M., \& Bressoud, E. (2008). L'acceptabilité du placement de marques dans les jeux vidéo: Une application aux joueurs de 15 à 35 ans. Presented in the 24th Congrès international de l'Association Française du Marketing, Paris, France.

Mackay, T., Ewing, M., Newton, F., \& Windisch, L. (2009). The effect of product placement in computer games on brand attitude and recall. International Journal of Advertising, 28(3), 423-438.

Mediative. (2014). The Evolution of Google's Search Results Pages \& Effects on User Behaviour. Resource document. Retrieved July 10, 2018, from http://www.mediative.com/fr/evolution-despages-de-resultats-de-recherche-de-google-et-les-effets-sur-les-utilisateurs/

Nelson, M. R. (2002). Recall of brand placements in computer/video games. Journal of Advertising Research, 42(2), 80-92.

Nelson, M. R., Keum, H., \& Yaros, R. A. (2004). Advertainment or adcreep game players' attitudes toward advertising and product placements in computer games. Journal of Interactive Advertising, 5(1), 3-21.

Nielsen. (2017). Traditional TV viewing trends 18-24 year olds. Retrieved July 10, 2018, from https://www.marketingcharts.com/featured-24817/attachment/nielsen-traditional-tv-viewingtrends-18-24-yo-q12011-q22017-dec2017

Nunnally, J. (1978). Psychometric Methods (pp. 464-465). McGraw-Hill, New York.

Pernice, K., \& Nielsen, J. (2009). How to Conduct Eyetracking Studies. Fremont, CA: Nielsen Norman Group.

Quinn, S., Stark, P., \& Edmunds, R. (2007). Eyetracking the news. In J. Moos (Ed.). The Poynter Institute.

Reeves, B., Lang, A., Kim, E. Y., \& Tatar, D. (1999). The effects of screen size and message content on attention and arousal. Media Psychology, 1(1), 49-67.

Robison, J. (2014). Mobile commerce vs. desktop: 7 differences. Retrieved July 10, 2018, from http://www.practicalecommerce.com/articles/75772-Mobile-Commerce-vs-Desktop-7Differences

Russell, C.A. (2002). Investigating the effectiveness of product placements in television shows: The role of modality and plot connection congruence on brand memory and attitude. Journal of Consumer Research, 29(3), 306-318.

Sajjacholapunt, P., \& Ball, L. J. (2014). The influence of banner advertisements on attention and memory: Human faces with averted gaze can enhance advertising effectiveness. Frontiers in Psychology. https://doi.org/10.3389/fpsyg.2014.00166.

Schneider, L. P., Systems, B., \& Cornwell, T. B. (2005). Cashing in on crashes via brand placement in computer games: The effects of experience and flow on memory. International Journal of Advertising, 24(3), 321-343.

Segal, B. (2018). Global Digital Future in Focus (Canada Edition). Retrieved July 10, 2018, from https://www.comscore.com/Insights/Presentations-and-Whitepapers/2018/Global-Digital-Futurein-Focus-2018-Canada-Edition 
Shimp, T. A. (1981). Attitude toward the ad as a mediator of consumer brand choice. Journal of Advertising, 10(2), 9-48.

Sjöblom, M., Törhönen, M. Hamari, J., \& Macey, J. (2019). The ingredients of Twitch streaming: Affordances of game streams. Computers in Human Behavior, 92, 20-28.

Solomon, M. R., White, K., \& Dahl D. W. (2013). Consumer Behaviour: Buying, Having, Being (6e éd.). Toronto: Pearson Canada.

Spears, N., \& Singh, S. N. (2004). Measuring attitude toward the brand and purchase intentions. Journal of Current Issues \& Research in Advertising, 26(2), 53-66.

Srivastava, R. K. (2015). Product placement by global brands as an alternative strategy: Is it worth in emerging market? Journal of Strategic Marketing, 23(2), 141-156.

Stern, B. B., \& Russell, C. A. (2004). Consumer responses to product placement in television sitcoms: Genre, sex, and consumption. Consumption Markets \& Culture, 7(4), 371-394.

Texeira, T. (2014). The rising cost of consumer attention: Why you should care, and what you can do about it. Harvard Business School Working Paper, pp. 14-55

Twitch. (2018). Annual report 2017: Year in review 2017. Retrieved December 15, 2018, from https://www.twitch.tv/year/2017/

Twitch. (2015). Annual report 2014: Two-thousands more teen. Retrieved July 10, 2018, from https://www.twitch.tv/year/2014

Twitch. (2017). Audience. Retrieved December 15, 2018, from http://twitchadvertising.tv/audience/

Verhellen, Y., Dens, N., \& De Pelsmacker, P. (2013). Consumer responses to brands placed in YouTube movies: The effect of prominence and endorser expertise. Journal of Electronic Commerce Research, 14(4), 287-303.

Williams, K., Petrosky, A., Hernandez, E., \& Page, R., Jr. (2011). Product placement effectiveness: Revisited and renewed. Journal of Management and Marketing Research, 7, 1-24.

Wolverton, G. S., \& Zola, D. (1983). The temporal characteristics of visual information extraction during reading. In K. Rayner (dir.), Eye Movements in Reading: Perceptual and Language Processes (pp. 41-51). New York, NY: Academic Press. 